\title{
Increased skeletal intermuscular fat is associated with reduced exercise capacity in cancer survivors: a cross-sectional study
}

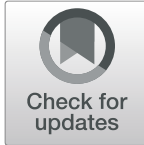

Kerryn W. Reding ${ }^{1,9}$, Peter Brubaker², Ralph D’Agostino $\mathrm{Jr}^{3}$, Dalane W. Kitzman ${ }^{4}$ Barbara Nicklas ${ }^{5}$, Dale Langford ${ }^{6}$, Michael Grodesky ${ }^{7}$ and W. Gregory Hundley ${ }^{4,8^{*}}$

\begin{abstract}
Background: Cancer survivors experience on average a $20 \%$ reduction in peak exercise capacity $\left(\mathrm{VO}_{2}\right.$ peak $)$ post-cancer treatment. Intermuscular fat (IMF) is a strong predictor of reduced exercise capacity in heart failure (HF) patients; however it is unknown whether increased IMF is related to reduced $\mathrm{VO}_{2}$ peak in cancer survivors.

Methods and results: Twenty eight individuals: 14 cancer survivors $>12$-months post-cancer treatment and 14 individuals without cancer were matched on age, gender, and body mass index (BMI). Participants underwent magnetic resonance imaging (MRI) assessments of IMF within the paraspinal muscles, $\mathrm{VO}_{2}$ peak and exerciseassociated measures of left ventricular ejection fraction (LVEF). Blinded analyses were performed. Associations between the ratio of IMF to skeletal muscle (SM) were estimated using Pearson's partial correlation coefficients. Individuals with cancer and non-cancer comparators were of similar age (54 \pm 17 versus $54 \pm 15$ years; $p=1.0)$, gender ( 5 men and 9 women, both groups), and $\mathrm{BMI}(27 \pm 4$ versus $26 \pm 4 ; p=0.57)$. Peak $\mathrm{VO}_{2}$ was $22 \%$ lower in cancer survivors versus non-cancer comparators (26.9 vs $34.3 \mathrm{ml} / \mathrm{kg} / \mathrm{min} ; p=0.005$ ), and was correlated with IMF:SM in both cancer survivors and non-cancer individuals after accounting for exercise-associated LVEF, resting LVEF, BMI, other body fat depots, and cardiovascular disease (CVD) co-morbidities ( $p<0.001$ to 0.08 for all adjusted correlations).

Conclusion: Among cancer survivors that previously received anthracyclines, increased intermuscular fat is associated with reduced $\mathrm{VO}_{2}$ peak even after accounting for exercise-associated cardiac function. This suggests IMF is important in the development of exercise intolerance, an outcome experienced by a large number of cancer survivors.
\end{abstract}

Keywords: Exercise intolerance, Muscle composition, Cancer survivors

\section{Introduction}

Peak exercise capacity $\left(\mathrm{VO}_{2}\right.$ peak $)$ is reduced by an average $20 \%$ among cancer survivors who received potentially cardiotoxic treatment for their cancer [1]. According to the Fick equation, $\mathrm{VO}_{2}$ peak is a function of cardiac output and the arterio-venous oxygen $\left(\mathrm{a}-\mathrm{VO}_{2}\right)$ difference, a measure indicating the ability of the periphery to extract oxygen from the circulating blood [2]. Given the association between anthracycline-based

\footnotetext{
* Correspondence: Greg.Hundley@vcuhealth.org

${ }^{4}$ Department of Internal Medicine (Section on Cardiology), Wake Forest

School of Medicine, Medical Center Boulevard, Winston-Salem, NC

27157-1045, USA

${ }^{8}$ Pauley Heart Center, Virginia Commonwealth University Health System,

Richmond, VA, USA

Full list of author information is available at the end of the article
}

chemotherapy and reduced left ventricular ejection fraction (LVEF), research in cancer survivors has predominantly focused on the role of cardiac dysfunction in reduced exercise capacity as opposed to factors that may impact the a-VO2 difference.

However, in non-cancer populations reductions in the exercise-associated a-VO2 difference predicts reduced $\mathrm{VO}_{2}$ peak [3]. Adipose tissue, particularly intermuscular fat (IMF), is metabolically active and competes with skeletal muscle for tissue perfusion and oxygen consumption $[4,5]$. A study in heart failure patients supported this notion, showing that the ratio of intermuscular fat (IMF) to skeletal muscle $(\mathrm{SM})$ predicted reductions in $\mathrm{VO}_{2 \text { peak }}$ [5]. Moreover, emerging data shows that IMF accumulates during cancer treatment [5]. The finding that IMF 
increases over the same period during which exercise capacity is reduced led us to evaluate the relationship between the IMF:SM ratio in paraspinal skeletal muscles and $\mathrm{VO}_{2}$ peak in cancer survivors.

\section{Methods}

We enrolled 14 cancer survivors $>12$-months after receipt of anthracycline-based chemotherapy, who were matched on age, gender, and body mass index (BMI) to 14 individuals without cancer serving as non-cancer comparators. This matching ensured that cancer survivors and non-cancer comparators were $<1$ unit apart on BMI and age, and that the range of values was within 1 and 2 units, respectively. Results on these participants have been presented in an International forum with the Society for Cardiovascular Magnetic Resonance [6]. Cancer survivors were identified from the hematology and oncology clinics at the Comprehensive Cancer Center at Wake Forest Health Sciences in Winston-Salem, North Carolina. The cancer survivors and non-cancer comparators had no prior myocardial infarction, heart failure, or other prior cardiovascular event. The exclusion criteria for cancer survivors and non-cancer comparators were contraindication for magnetic resonance imaging (MRI), including a pacemaker, defibrillator, or other implanted electronic devices; inability to perform exercise treadmill testing; pregnancy; claustrophobia; acute illness or injury related to walking briskly or running on a treadmill; or incapability to provide informed consent. All participants provided informed consent. This study was approved by the Institutional Review Board of the Wake Forest University School of Medicine.

According to previously published techniques [7], all participants underwent abdominal MRI with determination of IMF and SM in the paraspinal muscles, and subcutaneous, intraperitoneal, and retroperitoneal fat. Images were acquired with an axial non-contrast T1 weighted MRI scan positioned at the level of the second lumber vertebra (L2) using a 5-mm thick slice, a $256 \times$ 256 matrix, and a $180^{\circ}$ flip angle. TomoVision SliceOmatic version 5.0 was used to measure muscle and adipose tissue depots (Fig. 1). A MRI analyst manually separated muscle and fat using reproducible, previously described methods [5]. Briefly, the area of each adipose and muscle compartment was calculated as number of pixels multiplied by pixel surface area. IMF was defined as the adipose tissue visible by MRI within the boundary of the muscle fascia.

At the time of IMF acquisition, LVEF was measured from a series of cine white blood images $(8 \mathrm{~mm}$ thick with $2 \mathrm{~mm}$ gap, temporal resolution $40 \mathrm{msec}$, field of view $36 \mathrm{~cm}$, and $256 \times 128$ matrix) spanning the left ventricular (LV) base to apex acquired during 6 to 8 -s of breath-holding. LV volumes were determined using a modified Simpson's rule calculation, and the LVEF was determined by subtracting the LV end-systolic volume from the LV end-diastolic volume and dividing by the LV end-diastolic volume [7]. Images were analyzed by individuals blinded to all participant characteristics and other testing results.

After acquiring images to measure LV function at rest and paraspinal fat, each participant underwent a cardiopulmonary exercise treadmill stress test (CPET) measuring $\mathrm{VO}_{2}$ peak. For this, participants performed a treadmill test according to the Bruce protocol or modified Bruce protocol (depending on fitness level) to the point of maximal exercise as identified by trained staff who were blinded to their survivorship or non-cancer status. Immediately after attaining peak exercise, participants transferred back to the scanner and underwent acquisition of LV function within $50 \mathrm{~s}$ of exercise cessation, henceforth called exercise-associated LVEF.

Prior to MRI, each participant completed the Godin Leisure Time Physical Activity Questionnaire to ascertain self-reported physical activity [8]. This questionnaire measures the frequency of strenuous, moderate, and mild exercise performed during a typical 7 day period and is reported in times per week of exercise $>15 \mathrm{~min}$ in duration. Participants also completed the functional assessment of cancer therapy: fatigue (FACT-F) questionnaire, a 13-item questionnaire developed to assess fatigue in cancer patients over the last seven days [9]. Scores range from zero to 52 with a higher score indicating lower fatigue levels. A categorical variable indicating the number of CVD co-morbidities was created based on the presence of a) coronary artery disease, b) diabetes, and c) hypertension (using resting blood pressure and current use of antihypertensive medications).

Control and cancer patient groups were compared on baseline characteristics using 2-sample t-tests for continuous measures and Fisher's exact tests for binary measures. Next, Pearson correlation coefficients were estimated to examine the correlation between peak $\mathrm{VO}_{2}$ measures and intermuscular fat to skeletal muscle ratio separately for control and cancer patients. These correlations were estimated unadjusted, and adjusted individually for resting LVEF, exercise-associated LVEF, BMI, subcutaneous fat, and intraperitoneal fat.

\section{Results}

Five men and nine women were included in each group. Cancer survivors and controls were of similar age (54 \pm 17 and $54 \pm 15$ years) and BMI (27 \pm 4 and $26 \pm 4)$, respectively. Eight and six of the cancer survivors had breast cancer and lymphoma, respectively, with an average cumulative received dose of $327 \pm 139 \mathrm{mg} / \mathrm{m}^{2}$ in doxorubicin equivalents [10]. The proportion of participants with CVD co-morbidities was similar across the 


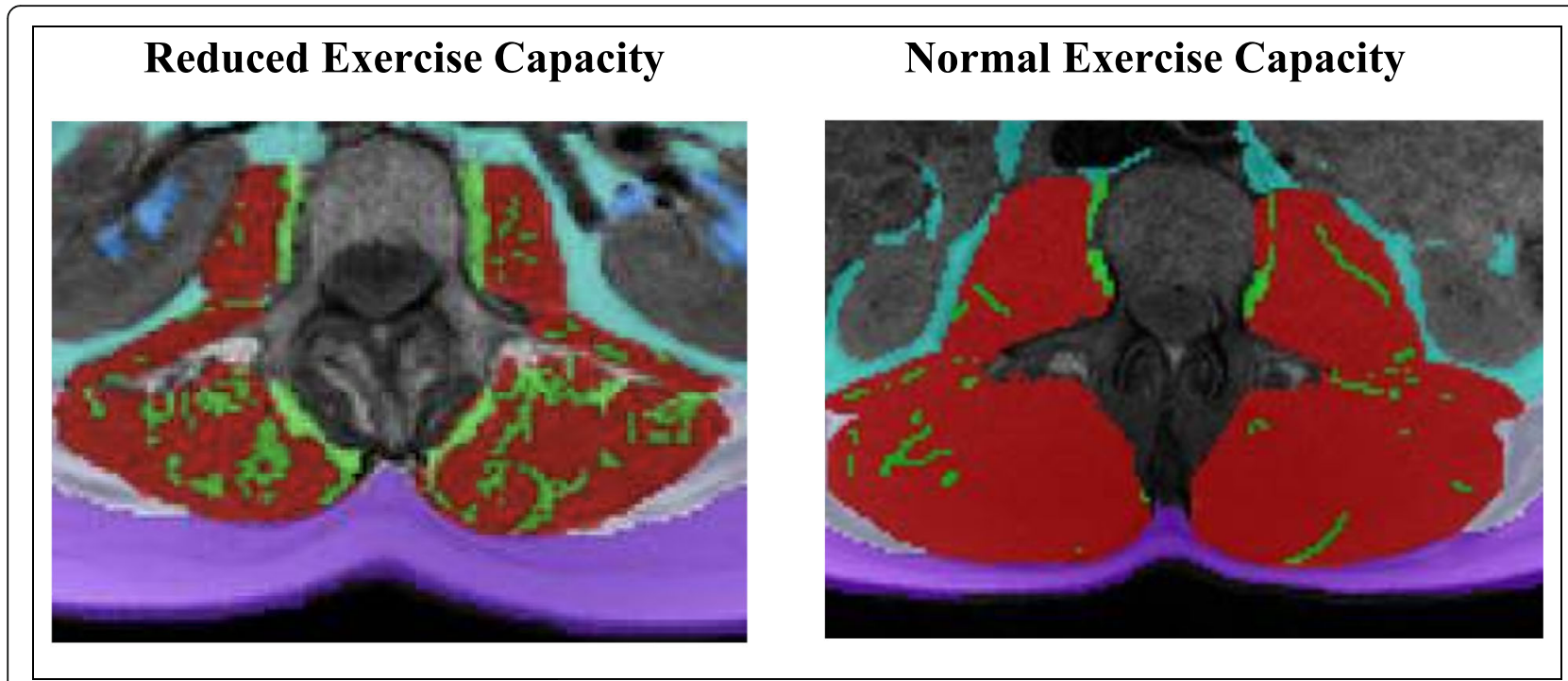

Fig. 1 The intermuscular fat of paraspinal muscles, depicted from MRI images of increased IMF (green) relative to SM (red) in a cancer survivor with reduced exercise capacity (left) and a control participant with normal exercise capacity (right)

two groups, with six cancer survivors and four non-cancer comparators reporting $1+$ CVD co-morbidity $(p=0.20)$. Cancer survivors and non-cancer comparators were similar with respect to self-reported fatigue $(46 \pm 6$ and $48 \pm 3$, respectively; $p=0.28)$ and times per week of engagement in mild $(3.5 \pm 2.5$ and $3.4 \pm 3.0$, respectively; $p=0.91)$ and moderate exercise $(3.4 \pm 2.4$ and $2.0 \pm 1.8$, respectively $p=0.12$ ), but differed in frequency of self-reported strenuous physical activity $(1.5 \pm 1.9$ and $3.8 \pm 2.0$, respectively; $p<0.01)$. Cancer survivors post-anthracycline-based treatment had marginally lower resting LVEF (53 \pm 6 and $57 \pm 7$, respectively; $p=0.07)$ and exercise-associated LVEF $(61 \pm 9$ and $67 \pm 8$, respectively; $p=0.09$ ) compared to non-cancer comparators.

$\mathrm{VO}_{2}$ peak was $22 \%$ lower in cancer survivors versus non-cancer comparators $(25.7 \pm 7$ and $34.3 \pm 10.3 \mathrm{ml} / \mathrm{kg} /$ min, respectively; $p=0.005)$. Compared to controls, cancer survivors trended toward more paraspinal IMF $\left(13.9 \pm 5.6\right.$ and $11.7 \pm 4.3 \mathrm{~cm}^{2}$, respectively; $\left.p=0.11\right)$ and a higher IMF:SM ratio $(0.26 \pm 0.10$ and $0.22 \pm 0.10$, respectively; $p=0.13)$. Among all participants, $\mathrm{VO}_{2}$ peak was inversely correlated with IMF:SM $(p<0.001)$, and persisted after adjustment for LVEF, CVD-comorbidities, and other depots of adipose tissue $(p<0.0001$ to 0.007; Table 1). These inverse correlations were seen in cancer survivors and non-cancer comparators separately $(\mathrm{r}=-$ $0.545, p=0.04 ; \mathrm{r}=-0.721, p=0.004$, respectively). The correlations between IMF:SM and $\mathrm{VO}_{2}$ peak in both cancer survivors and non-cancer comparators persisted after adjustment for the same variables, as correlations remained above 0.50 for cancer survivors and above 0.59 for non-cancer comparators. Specifically, the correlations between IMF:SM and $\mathrm{VO}_{2}$ peak remained after adjustment for resting $\operatorname{LVEF}(\mathrm{p}=0.04$; $p=0.004$, respectively), for exercise-associated LVEF $(p=0.06 ; p<0.001$, respectively), and for CVD risk factors ( $p=0.08 ; p=0.003$, respectively). The correlations also persisted after accounting for body composition measures of BMI ( $\mathrm{p}=0.04 ; p=0.007$, respectively), intraperitoneal fat ( $p=0.05 ; p=0.03$, respectively); and subcutaneous fat $(p=0.08 ; p=0.008$, respectively).

\section{Discussion}

To our knowledge, this is the first report that IMF:SM is correlated with reduced $\mathrm{VO}_{2}$ peak in cancer survivors. This finding occurred in the context of a study showing reduced exercise capacity in cancer survivors versus matched non-cancer comparators, with marginal differences in resting and exercise-associated LVEF. In addition, we observed a marginal difference in IMF in cancer survivors versus non-cancer comparators. Taken together, our findings suggest that IMF:SM as well as potential decrements in LVEF, may contribute to reduced exercise capacity in cancer survivors. This concept of involvement of both skeletal muscle and cardiovascular factors in reduced exercise capacity is consistent with the literature in exercise intolerance of heart failure with preserved ejection fraction (HFpEF) and the literature in exercise capacity after cancer treatment [11, 12]. Our finding of a correlation between IMF:SM and exercise intolerance is strengthened by results showing that the relationship between IMF:SM and $\mathrm{VO}_{2}$ peak was not attenuated by adjustment for visceral fat, CVD co-morbidities, or by resting LVEF or immediately post-exercise measurements of LVEF. 
Table 1 Correlations of $\mathrm{VO}_{2}$ peak with IMF:SM in cancer survivors and non-cancer comparators

\begin{tabular}{|c|c|c|c|c|c|c|}
\hline \multirow{3}{*}{ Adjusted for: } & \multicolumn{6}{|c|}{ IMF:SM correlation with $\mathrm{VO}_{2}$ peak } \\
\hline & \multicolumn{2}{|c|}{$\begin{array}{l}\text { Overall study population } \\
(n=28)\end{array}$} & \multicolumn{2}{|c|}{$\begin{array}{l}\text { Cancer Survivors } \\
(n=14)\end{array}$} & \multicolumn{2}{|c|}{$\begin{array}{l}\text { Non-cancer comparators } \\
(n=14)\end{array}$} \\
\hline & $r$ & $p$-value & $r$ & $p$-value & $r$ & $p$-value \\
\hline$N / A^{a}$ & -0.67 & 0.0001 & -0.54 & 0.044 & -0.72 & 0.004 \\
\hline Resting LVEF \% & -0.70 & $<0.0001$ & -0.59 & 0.035 & -0.74 & 0.004 \\
\hline Exercise-associated LVEF \% & -0.71 & $<0.0001$ & -0.54 & 0.055 & -0.85 & $<0.001$ \\
\hline CVD risk factors & -0.64 & 0.0003 & -0.50 & 0.083 & -0.75 & 0.003 \\
\hline BMI & -0.67 & 0.001 & -0.58 & 0.040 & -0.71 & 0.007 \\
\hline SC fat & -0.61 & 0.007 & -0.50 & 0.080 & -0.70 & 0.008 \\
\hline IP fat & -0.60 & 0.001 & -0.56 & 0.047 & -0.59 & 0.033 \\
\hline
\end{tabular}

${ }^{\mathrm{a}} \mathrm{N} / \mathrm{A}$ : Correlation is not adjusted for any factors

LVEF left ventricular ejection fraction, IMF intermuscular fat, $S M$ skeletal muscle, $B M I$ body mass index, SC subcutaneous, IP intraperitoneal

Prior studies have reported findings of an inverse correlation between IMF:SM and exercise capacity in non-cancer populations, similar to what our study observed. Notably, in $38 \mathrm{HFpEF}$ patients, thigh IMF:SM was the strongest predictor of reduced $\mathrm{VO}_{2 \text { peak }}$ of all body composition factors investigated, including subcutaneous fat, total thigh area, and thigh compartment area [5]. Our study focused on paraspinal IMF in the abdominal region because abdominal images are acquired frequently to stage a variety of cancers, and prior studies have used these images to assess cachexia [13]. In addition, the paraspinal muscles are well seen in abdominal MRI scans, along with depots of visceral and subcutaneous fat [14]. This allows one to distinguish ill effects of IMF relative to these other fat depots. Prior studies have shown that IMF has worse health impacts than subcutaneous fat potentially due to its role in impairing mitochondrial function, promoting muscle fibrosis, and competing with muscle for oxygen perfusion $[4,5]$.

While muscle wasting during cancer treatment has been appreciated for some time, less attention has been paid to changes in muscle composition. Two recent studies in cancer survivors have shown that muscle composition changes during chemotherapy. In 200 lung cancer patients, abdominal IMF increased by $7 \%$ after first-line chemotherapy in $(P<0.001)$ [15]. In 73 metastatic breast cancer patients treated with taxane-based chemotherapy, muscle attenuation decreased (indicating increased accumulation of IMF) during chemotherapy $(P=0.03)[16]$. The importance of IMF in cancer survivors was shown by a 2017 study reporting that muscle attenuation was the strongest risk factor (of all body composition measures) for increased mortality (Hazard Ratio $=2.0[1.3-3.1])$ in 166 metastatic breast cancer patients [17]. Thus, prior research provides support for an accumulation of IMF during treatment and its impact on survival.
One limitation of the current study is its small sample size. We attempted to mitigate the impact of a small sample through the design of our study. Namely, we performed matching of cancer survivors to non-cancer comparators on age, gender, and BMI, factors most likely to impact $\mathrm{VO}_{2}$ peak. This efficient design reduced potential bias by minimizing the impact of confounding in our study sample, and provided for more precise correlation estimates. A second limitation relates to the timing of ascertainment of our exposures and outcomes as we were unable to examine changes in IMF:SM and $\mathrm{VO}_{2}$ peak during cancer treatment. Future studies could assess whether IMF changes prior to, during, or after cancer treatment impact changes in $\mathrm{VO}_{2}$ peak. The change in IMF during cancer treatment is of particular interest due to the documented reduction in exercise capacity during this period in past studies $[1,18,19]$.

Strengths of our study include its use of MRI to ascertain IMF area which allows for a direct assessment of intermuscular fat as shown in Fig. 1, whereas computed tomography $(\mathrm{CT})$ scans calculate muscle attenuation which indirectly assesses IMF [20]. Another strength is the assessment of LVEF which allows for an examination of both cardiac and peripheral factors in relation to exercise capacity in cancer survivors compared to non-cancer comparators.

In summary, our findings suggest that IMF accumulation is an important contributor to reduced $\mathrm{VO}_{2}$ peak among cancer survivors and are in accord with findings in other disorders associated with reduced $\mathrm{VO}_{2}$ peak $[5,21]$. These findings suggest that skeletal muscle abnormalities, in addition to heart dysfunction, contribute to the profound reductions in exercise capacity in cancer survivors, and thus need to be evaluated. Should these findings be replicated, this would provide insight into the pathophysiology of the severe, persistent exercise intolerance in cancer survivors and provide novel therapeutic targets. 


\section{Abbreviations}

a- $\mathrm{VO}_{2}$ : arterio-venous oxygen; BMI: Body mass index; CPET: Cardiopulmonary exercise treadmill stress test; CVD: Cardiovascular disease; FACT-F: Functional assessment of cancer therapy- fatigue; HF: Heart failure; IMF: Intermuscular fat; L2: Second lumber vertebra; LV: Left ventricular; LVEF: Left ventricular ejection fraction; MRI: Magnetic resonance imaging; SM: Skeletal muscle; $\mathrm{VO}_{2}$ peak: peak exercise capacity

\section{Acknowledgments}

We wish to thank the study participants for their involvement in this study.

\section{Funding}

Research supported in part by National Institute of Health grants R01CA199167, R01CA167821, R01HL118740; NCI Cancer Center Support Grant (P30CA012197); and Hope Heart Institute provided fellowship support to K. Reding.

\section{Availability of data and materials}

The datasets analyzed during the current study are available from the corresponding author on reasonable request.

\section{Authors' contributions}

All authors have approved this manuscript for publication. KWR had a role in the analysis plan, interpretation of data, and manuscript writing; PB had a role in study conception, acquisition of data, analysis and interpretation of data; RD had a role in study conception, design, and analysis and interpretation of data; DWK, BN, DL, and MG had a role in interpretation of data and manuscript editing; WGH had a role in study conception, design, acquisition of data, analysis interpretation of data, and manuscript writing.

\section{Ethics approval and consent to participate}

This research involved human subjects and was performed in accordance with the Declaration of Helsinki. All participants provided informed consent. This study was approved by the Institutional Review Board of the Wake Forest University School of Medicine.

\section{Consent for publication}

Study participants agreed to have their data used in publications.

\section{Competing interests}

The authors have the following disclosures to declare: Dr. Kitzman has been a consultant for Relypsa, Abbvie, GlaxoSmithKline, AstraZeneca, Merck, CinRx, St. Luke's Medical Center, DCRI, and Corvia Medical; received grants from Novartis and St. Luke's Medical Center; and owns stock in Gilead Sciences.

\section{Publisher's Note}

Springer Nature remains neutral with regard to jurisdictional claims in published maps and institutional affiliations.

\section{Author details}

'Department of Biobehavioral Nursing and Health Informatics, University of Washington School of Nursing, Seattle, WA, USA. ${ }^{2}$ Department of Health and Exercise Science, Wake Forest University, Winston-Salem, NC, USA. ${ }^{3}$ Department of Biostatistical Sciences, Wake Forest School of Medicine, Winston-Salem, NC, USA. ${ }^{4}$ Department of Internal Medicine (Section on Cardiology), Wake Forest School of Medicine, Medical Center Boulevard, Winston-Salem, NC 27157-1045, USA. ${ }^{5}$ Gerontology and Geriatric Medicine, Wake Forest School of Medicine, Winston-Salem, NC, USA. ${ }^{6}$ Department of Anesthesiology and Pain Medicine, University of Washington School of Medicine, Seattle, WA, USA. ${ }^{7}$ Department of Psychosocial and Community Health, University of Washington School of Nursing, Seattle, WA, USA ${ }^{8}$ Pauley Heart Center, Virginia Commonwealth University Health System, Richmond, VA, USA. ${ }^{9}$ Department of Cancer Prevention, Public Health Sciences, Fred Hutch Cancer Research Center, Seattle, WA, USA
Received: 27 December 2018 Accepted: 17 April 2019

Published online: 03 May 2019

\section{References}

1. Jones LW, Haykowsky M, Pituskin EN, Jendzjowsky NG, Tomczak CR, Haennel RG, Mackey JR. Cardiovascular reserve and risk profile of postmenopausal women after chemoendocrine therapy for hormone receptor--positive operable breast cancer. Oncologist. 2007;12(10):1156-64 https://doi.org/10.1634/theoncologist.12-10-1156 PubMed PMID: 17962609.

2. Albouaini $K$, Egred M, Alahmar A, Wright DJ. Cardiopulmonary exercise testing and its application. Heart. 2007:93(10):1285-92. https://doi.org/10. 1136/hrt.2007.121558 PubMed PMID: 17890705; PubMed Central PMCID: PMC2000933.

3. Haykowsky MJ, Brubaker PH, John JM, Stewart KP, Morgan TM, Kitzman DW. Determinants of exercise intolerance in elderly heart failure patients with preserved ejection fraction. J Am Coll Cardiol. 2011;58(3):265-74. https://doi. org/10.1016/j.jacc.2011.02.055 PubMed PMID: 21737017; PubMed Central PMCID: PMC3272542.

4. Addison O, Marcus RL, Lastayo PC, Ryan AS. Intermuscular fat: a review of the consequences and causes. Int J Endocrinol. 2014:2014:309570. https:// doi.org/10.1155/2014/309570 PubMed PMID: 24527032; PubMed Central PMCID: PMC3910392

5. Haykowsky MJ, Kouba EJ, Brubaker PH, Nicklas BJ, Eggebeen J, Kitzman DW. Skeletal muscle composition and its relation to exercise intolerance in older patients with heart failure and preserved ejection fraction. Am J Cardiol. 2014:113(7):1211-6. https://doi.org/10.1016/j.amjcard.2013.12.031 PubMed PMID: 24507172; PubMed Central PMCID: PMC4282135.

6. Jordan J, Brubaker P, Jensen A, Reding KW, Lamar Z, Mihalko SL, Haykowsky M, Jones L, D'Agostino RB Jr, Kitzman DW, Hundley G. Exercise capacity is reduced in Cancer survivors previously treated with anthracycline-based chemotherapy despite a preserved cardiac output response. Proceedings from the 22nd annual SCMR scientific sessions; 2019. p. 986.

7. Vasu S, Morgan TM, Kitzman DW, Bertoni A, Stacey RB, Hamilton C, Chiles C, Thohan V, Hundley WG. Abnormal stress-related measures of arterial stiffness in middle-aged and elderly men and women with impaired fasting glucose at risk for a first episode of symptomatic heart failure. J Am Heart Assoc. 2015:4(1):e000991. https://doi.org/10.1161/JAHA.114.000991 PubMed PMID: 25589534; PubMed Central PMCID: PMC4330048.

8. Godin G, Shephard RJ. A simple method to assess exercise behavior in the community. Can J Appl Sport Sci. 1985;10(3):141-6 PubMed PMID: 4053261.

9. Functional Assessment of Cancer Therapy: Fatigue (FACT-F). [cited 2018]. Available from: http://qol.thoracic.org/sections/instruments/fj/ pages/fact-f.html.

10. Feijen EA, Leisenring WM, Stratton KL, Ness KK, van der Pal HJ, Caron HN, Armstrong GT, Green DM, Hudson MM, Oeffinger KC, Robison LL, Stovall M, Kremer LC, Chow EJ. Equivalence ratio for Daunorubicin to doxorubicin in relation to late heart failure in survivors of childhood Cancer. J Clin Oncol. 2015;33(32):3774-80. https://doi.org/10.1200/JCO. 2015.61.5187 PubMed PMID: 26304888; PubMed Central PMCID: PMC4737860

11. Kitzman DW, Shah SJ. The HFpEF obesity phenotype: the elephant in the room. J Am Coll Cardiol. 2016;68(2):200-3. https://doi.org/10.1016/j.jacc.2016 05.019 PubMed PMID: 27386774

12. Lakoski SG, Eves ND, Douglas PS, Jones LW. Exercise rehabilitation in patients with cancer. Nat Rev Clin Oncol. 2012;9(5):288-96. https://doi.org/ 10.1038/nrclinonc.2012.27 PubMed PMID: 22392097; PubMed Central PMCID: PMC3640332.

13. Penet MF, Winnard PT Jr, Jacobs MA, Bhujwalla ZM. Understanding cancerinduced cachexia: imaging the flame and its fuel. Curr Opin Support Palliat Care. 2011;5(4):327-33. https://doi.org/10.1097/SPC.0b013e32834c49ba PubMed PMID: 21986909; PubMed Central PMCID: PMC4155489.

14. Schweitzer L, Geisler C, Pourhassan M, Braun W, Gluer CC, Bosy-Westphal A, Muller MJ. What is the best reference site for a single MRI slice to assess whole-body skeletal muscle and adipose tissue volumes in healthy adults? Am J Clin Nutr. 2015:102(1):58-65. https://doi.org/10.3945/ajcn.115.111203 PubMed PMID: 26016860.

15. Nattenmuller J, Wochner R, Muley T, Steins M, Hummler S, Teucher B, Wiskemann J, Kauczor HU, Wielputz MO, Heussel CP. Prognostic impact of CT-quantified muscle and fat distribution before and after first-linechemotherapy in lung Cancer patients. PLoS One. 2017;12(1):e0169136. 
16. Rier HN, Jager A, Sleijfer S, van Rosmalen J, Kock M, Levin MD. Changes in body composition and muscle attenuation during taxane-based

chemotherapy in patients with metastatic breast cancer. Breast Cancer Res Treat. 2018;168(1):95-105. https://doi.org/10.1007/s10549-017-4574-0 PubMed PMID: 29147870

17. Rier HN, Jager A, Sleiffer S, van Rosmalen J, Kock M, Levin MD. Low muscle attenuation is a prognostic factor for survival in metastatic breast cancer patients treated with first line palliative chemotherapy. Breast. 2017;31:9-15. https://doi.org/10.1016/j.breast.2016.10.014 PubMed PMID: 27810702.

18. Haykowsky MJ, Scott JM, Hudson K, Denduluri N. Lifestyle Interventions to Improve Cardiorespiratory Fitness and Reduce Breast Cancer Recurrence. Am Soc Clin Oncol Educ Book Meet. 2017;37:57-64. https://doi.org/10 14694/EDBK_175349 PubMed PMID: 28561685.

19. Haykowsky MJ, Beaudry R, Brothers RM, Nelson MD, Sarma S, La Gerche A. Pathophysiology of exercise intolerance in breast cancer survivors with preserved left ventricular ejection fraction. Clin Sci (Lond). 2016;130(24): 2239-44. https://doi.org/10.1042/CS20160479 PubMed PMID: 27815508.

20. McGregor RA, Cameron-Smith D, Poppitt SD. It is not just muscle mass: a review of muscle quality, composition and metabolism during ageing as determinants of muscle function and mobility in later life. Longev Healthspan. 2014;3(1):9. https://doi.org/10.1186/2046-2395-3-9 PubMed PMID: 25520782; PubMed Central PMCID: PMC4268803.

21. Beavers KM, Beavers DP, Houston DK, Harris TB, Hue TF, Koster A, Newman AB, Simonsick EM, Studenski SA, Nicklas BJ, Kritchevsky SB. Associations between body composition and gait-speed decline: results from the health, aging, and body composition study. Am J Clin Nutr. 2013;97(3):552-60. https://doi.org/10.3945/ajcn.112.047860 PubMed PMID: 23364001; PubMed Central PMCID: PMC3578402

Ready to submit your research? Choose BMC and benefit from:

- fast, convenient online submission

- thorough peer review by experienced researchers in your field

- rapid publication on acceptance

- support for research data, including large and complex data types

- gold Open Access which fosters wider collaboration and increased citations

- maximum visibility for your research: over $100 \mathrm{M}$ website views per year

At $\mathrm{BMC}$, research is always in progress.

Learn more biomedcentral.com/submissions 